\title{
Links Between Surgical Landmarks of the Temporal Bone and Cochlear Implant Approaches
}

\author{
1 Department of Otorhinolaryngology, University of Medicine and Pharmacy, Tîrgu-Mureș, Romania \\ 2 Department of Radiology, Emergency County Hospital Tîrgu-Mureș, Romania \\ ${ }^{3}$ Department of Forensic Medicine, University of Medicine and Pharmacy, Tîrgu-Mureș, Romania \\ ${ }^{4}$ Department of Human Anatomy, University of Medicine and Pharmacy, Tîrgu-Mureș, Romania
}

Lostun Gabriel ${ }^{1 *}$, Gușavan Diana Elena², Genes lonela1', Lostun Alexandra³ ${ }^{3}$ Brînzaniuc Klara4

Objective: We aimed to underline the surgical importance of the distances between the landmarks of the temporal bone, important for quantifying the benefits and disadvantages of two different cochlear implant techniques.

Methods: We have gathered all data from the Radiology Department in Emergency County Hospital in Tîrgu Mureș, namely computed tomography imagistic studies in order to perform the required measurements, according to pre-defined inclusion/exclusion criteria. The time interval was 5 months.

Results: The comparison between the sets of data shows a good match for the risk/benefit ratio for the two types of technique for cochlear implantation.

Conclusions: The middle cerebral fossa approach for the electrode insertion into the cochlea is a viable and needed surgical technique as the classic approach has reached its boundaries and new challenges appear. As surgical decisions are largely based on radiology data, our work underlines the importance of solving the borderline pathology, the extreme cases and the role of surgery in improving the quality of life for every patient with cochlear implant indication.

Keywords: temporal bone, cochlear implant, computed tomography, cochlea, eminentia arcuata

Received: 09 December 2014 / Accepted 04 June 2015

\section{Introduction}

In every specialty we have a borderline pathology. For Otorhinolaryngology such a borderline pathology is represented by Neurotology. The advances in every day medicine brought us closer to the necessity of solving extreme cases as many of the regular illnesses have found their cures. Even if Otology has started the development of its modern era a bit later, when technical support such as performant microscopes was invented, it grew with a high speed. In a couple of decades the first attempts for cochlear implantation were realized and nowadays these techniques help people of all ages worldwide to regain their hearing sense.

Advances in hearing rehabilitation by implantable prosthesis were reached step by step when Bone Anchored Hearing Aid, Partial or Total Middle Ear Prosthesis, Vibrant Soundbridge and Auditory Brainstem Implant were developed as medical technologies [1].

None of the above mentioned medical breakouts would not have been possible unless every little anatomical detail was known. Anatomia clavis medicinae is an old saying meaning the anatomy is the hand, the tool by which Medicine works. In order to reach this desiderate in some points it was necessary for the help of another branch - Radiology.

We consider important for the development of Medicine and Surgery to always try to find alternate pathways in

* Correspondence to: Gabriel Lostun

E-mail: gabriellostun@gmail.com order to help people, cure diseases, and provide solutions for every special case.

While a surgical technique such as cochlear implantation addresses a wide age range of patients it is not very rare for the surgeon to find himself facing anatomical variations and associated pathologies that bring up a question mark towards alternate approaches for the cochlear insertion of the electrode. Our goal through this article was to assess the risk and benefits from an anatomic and radiologic point of view and to compare the classic mastoid and middle cerebral fossa approach of the basal cochlear turn [2-4].

\section{Material and method}

For our study we have gathered in the first part radiologic data from the Radiology Clinic in the Emergency County Hospital in Tîrgu Mureș. For five months we examined and counted the computed tomography studies in this clinic that had as subject the head. The tomography tool used for our studies was Siemens Somatom Definiton AS, and Syngo Fastview was the software for applying 3D reconstruction protocols in order to obtain the wanted inner ear sections. We excluded from our statistics all the data that were modified by various artifacts such as incorrect examination given by movement of the patient, asymmetry between left and right side, image modification due to dental materials or other foreign objects. Sections were set to $0,6 \mathrm{~mm}$ as the inner ear protocol requests. We used coronal and axial sections. The head tomography studies 
were performed for various acute or chronic conditions, medical or surgical, and the trauma pathology of the head and neck was excluded as it may influence our data. We did not follow the usage or not of contrast substance, as we only worked with bone tissue measurements. All the cases that underwent surgery for skull base, inner or middle ear, were excluded from the study.

We first wanted to assess the presence of some important anatomic and surgical landmarks: eminentia arcuata, petro-tympanic suture and the great petrous superficial nerve and its canal. We searched for facial nerve canal abnormalities such as dehiscence, anatomic variations of the position of the jugular vein sinus and the degree of pneumatisation of the mastoid bone.

For correct appreciation of the risks and benefits of the two cochlear implant approaches for insertion of the electrode we measured the following distances: between round window and facial nerve recess, oval window, carotid canal, jugular foramen. The thickness of the bone above the basal turn of the cochlea was also quantified.

In the second part of the study the numbers obtained through our study, as described above, were used in order to sustain the importance and viability of the alternate approach of the basal turn of the cochlea, through the middle cranial fossa.

\section{Results}

For the given time interval -5 months -497 head computed tomography were included in our study, which meant 994 temporal bones underwent inner ear protocol and $3 \mathrm{D}$ reconstruction in order to obtain the axial and coronal sections.

The surgical landmarks eminentia arcuata, greater superficial petrous nerve canal and petro-tympanic suture were all found in every patient included in our study, on both temporal bones upper surface. All of the temporal bones examined presented normal pneumatisation of the mastoid part. In our research we have found no facial nerve canal or middle cerebral fossa dehiscence, while the jugular vein sinus presented no abnormalities.

The first distances important to be measured were between the round window and facial nerve recess, jugular foramen, carotid artery canal respectively oval window (Figure 1-4, Table I).

For the distance between the round window and facial nerve recess the variation was between 4,9 millimeters and 7,3 millimeters, measured on coronal sections. The aver-

Table I. The average and extreme values for the distances measured from the round window to chosen surrounding structures

\begin{tabular}{lcccc}
\hline & 1 & 2 & 3 & 4 \\
\hline Average & 5,84 & 4,30 & 3,38 & 9,36 \\
Highest value & 7,3 & 6,9 & 3,9 & 13,4 \\
Lowest value & 4,9 & 3,2 & 2,4 & 5,6 \\
\hline
\end{tabular}

1 - distance between round window and facial nerve recess

2 - distance between round window and jugular foramen

3 - distance between round window and oval window

4 - distance between round window and carotid artery canal

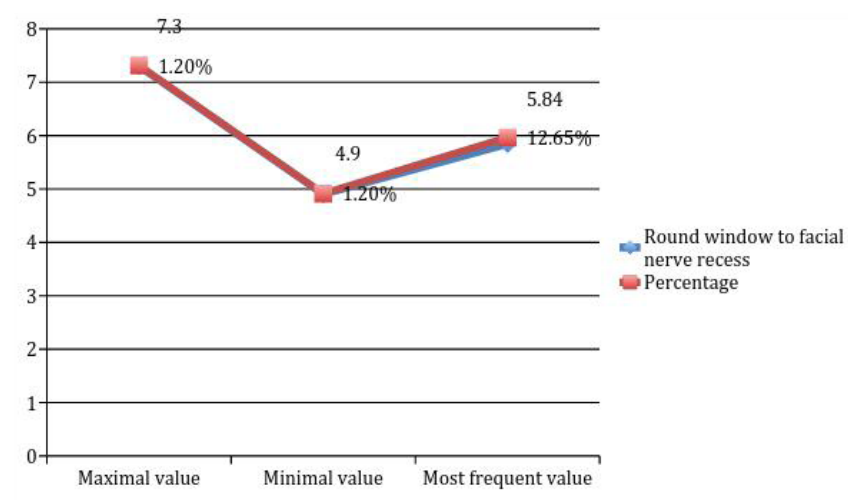

Fig. 1. Round window to facial nerve recess - relationship between maximal, minimal and most frequent value and their percentages

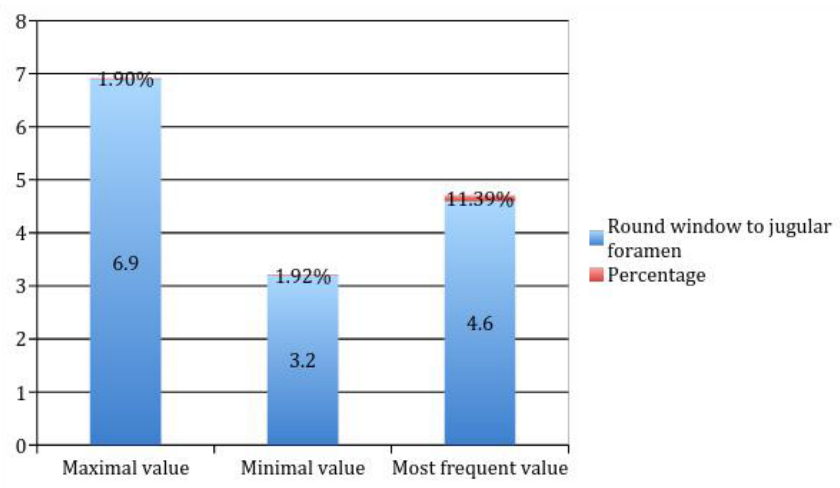

Fig. 2. Round window to jugular foramen - links from important values to percentage

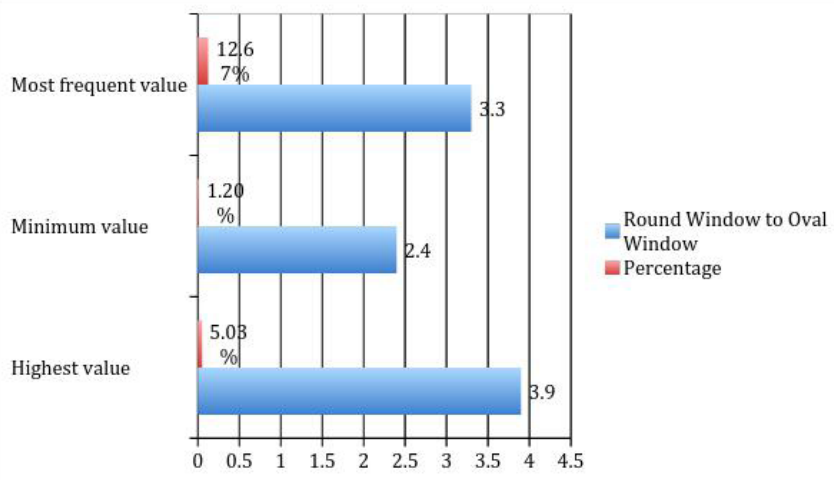

Fig. 3. Round window to oval window - percentages of the mean values

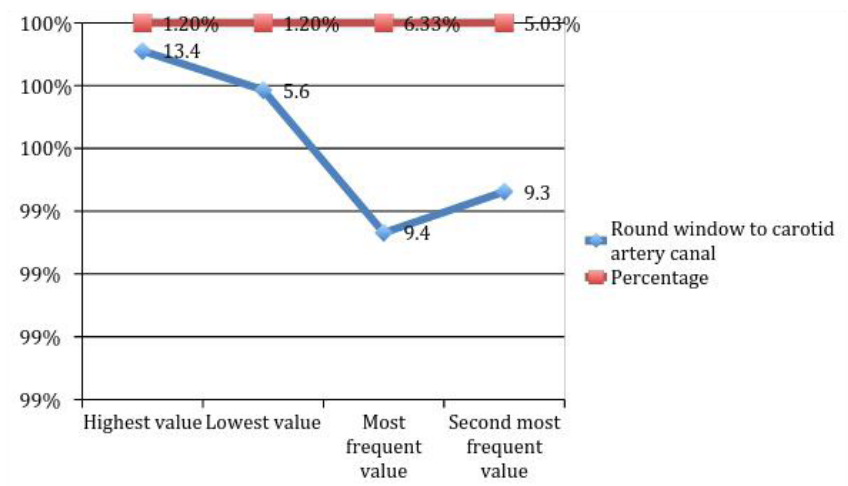

Fig. 4. Round window to carotid artery canal - relation between percentages of various values 
age was $5.84 \mathrm{~mm}$. The maximum value was met 12 times meaning a percentage of $1.20 \%$ and the minimal one the same. The most frequent value was $5.9 \mathrm{~mm}$, repeated 125 times $(12.65 \%)$.

The axis between round window and jugular foramen had extreme values of 3.2 millimeters and 6.9 millimeters, on axial sections. The average was $4.30 \mathrm{~mm}$. The extreme values represented less than $2 \%$ each. The most frequent value was $4.6 \mathrm{~mm}$, found 113 times (11.39\%).

The line from the round window to oval window had a minimal value of $2.4 \mathrm{~mm}$ and a maximal one of $3.9 \mathrm{~mm}$, as counted on coronal sections. The average was $3.38 \mathrm{~mm}$. The highest value was met 50 times meaning $5.03 \%$ while the smallest was again $1.20 \%$. The most frequent value was $3.3 \mathrm{~mm}$, met 126 times (12.67\%).

The length between round window and carotid artery canal had variations from $5.6 \mathrm{~mm}(1.20 \%)$ to $13.4 \mathrm{~mm}$ (1.20\% of the total number of measurements) on axial sections. The average was $9.36 \mathrm{~mm}$. The most frequent distance was $9.4 \mathrm{~mm}-63$ cases $(6.33 \%)$, followed shortly by $9.3 \mathrm{~mm}-50$ cases $(5.03 \%)$.

The thickness of the bone of the skull base covering the region above the basal turn of the cochlea was found to have variations between $5.9 \mathrm{~mm}$ and $9.2 \mathrm{~mm}$, both in small percentage of just over $1 \%(1.20 \%)$. The average was $7.40 \mathrm{~mm}$.

Variations were found as normal accompanying the age of the individuals, as subjects between 2 years old and 85 years old were included in the study. Yet a variation was found between individuals of the same age decade as the mastoid bone suffers a different degree of development and this strongly influences the anatomical distances between the above-mentioned landmarks. No difference was met between the two mastoid bones and temporal bone structures of the same subject.

\section{Discussions}

The fact that the anatomical landmarks that we searched on the upper surface of the middle cerebral fossa were found in every patient examined and bilaterally shows their reliability for the surgical technique. Other authors also measured the angles described by the corners of the triangle, therefore we did not consider necessary to repeat those measurements [5].

Every temporal bone examined presented a normal development of the mastoid part and no anatomic abnormalities were found, this proving ideal situation is quite often met in practice and for this the classic electrode insertion technique is suitable [6-10].

The average bone thickness above the basal turn of the cochlea can be considered quite safe as we are talking about $0.6 \mathrm{~mm}$ burr heads for cochleostomy and we have found a $7.4 \mathrm{~mm}$ value.

The distances measured in our study showed a large variability and a large area of dispersion for the values, similar to the real life [11-15].
No hierarchy was created in relation with age and sex as the authors have considered it useless. Nowadays the children have a slight priority because of their age related neural plasticity, however the extension for cochlear implantation grows day by day.

\section{Conclusions}

The data we have gathered present a high statistical significance, as many of the studies on temporal bones include numbers such as dozens and we nearly reached 1000 .

The anatomic landmarks searched for sustaining the alternate technique for electrode insertion in the basal turn of the cochlea are reliable. As they are always present and a correlation between them was found by other authors in a manner of angles (as they describe a triangle) we consider them as the ,anatomic pillars,, for this new technique.

Normal and safe to drill bone thickness above the basal turn of the cochlea is another strong argument for the alternate technique using the middle cerebral fossa approach.

The large difference among the extreme numbers regarding the distances measured between the round window and noble anatomic structures in the area gives a major uncertainty for the safeness of every surgical act. We might find ourselves in a critical or ideal situation every time. The fact that the smallest number for a distance can be 2 or 3 times smaller than the highest one can turn out quite challenging for the surgeon.

Some of the distances measured could not be considered risky indeed but when the success depends on many variables and concurrence of more than one factor may appear in an individual we strongly not advise to take the risk.

However, the observations that every temporal bone examined had normal mastoid part cellular development and no anatomic variation was found for the facial nerve canal sustains some security also for the basic technique $[16,17,18]$.

The technique recommended by the authors has pro and contra arguments as any other. We believe that omniscient anatomic landmarks and safe bone thickness are a very good foundation for it. Our work has continuity with other studies presenting the angles of the triangle described by these structures and the papers sustain each other.

The authors will continue their research by applying practically the sustained theory through the study of the learning curve for this alternate approach in $3 \mathrm{D}$ reconstructed anatomic models, based on the real cases analyzed in this work.

The aim of anatomy and radiology is to ease up the work of the surgeon and not raise too many question marks in his head. Therefore he should not be faced with too many anatomic variations, instead he should have safe and quick solutions.

The middle cerebral fossa approach for the electrode insertion into the cochlea is a viable and needed surgical technique as the classic approach has reached its boundaries and new challenges appear. As any good surgeon puts 
a great accent on the radiology data, our work is meant to give more power and confidence to the ones thinking about solving the borderline pathology, the extreme cases and helping improve the quality of life for every patient with cochlear implant indication.

Anatomy, surgery and in general medicine will always look for alternate pathways in order to reach their goal, to offer solution for everyone no matter we are discussing a case in a million or a majority.

\section{Acknowledgements}

This paper was published under the framework of European Social Found, Human Resources Development Operational Programme 2007-2013, project no. POSDRU/159/1.5/S/136893

\section{References}

1. Bishop CE, Eby TL. The current status of audiologic rehabilitation for profound unilateral sensorineural hearing loss. Laryngoscope. 2010;120:552-556.

2. Brito RD, Bittencourt AG, Tsuji RK, Magnan J, Bento RF. Cochlear implantation through the middle fossa: an anatomic study for a novel technique. Acta Otolaryngol. 2013;133(9):905-909.

3. Handzel $\mathrm{O}$, Wang $\mathrm{H}$, Fiering $\mathrm{J}$, et al. Mastoid cavity dimensions and shape: method of measurement and virtual fitting of implantable devices. Audiol Neurootol. 2009;14(5):308-314.

4. Todd NW. Cochlear implantation via the middle fossa: surgical and electrode array considerations. Cochlear Implants Int. 2007;8(1):12-28.

5. Seo Y, Ito T, Sasaki T, Nakagawara J, Nakamura H. Assessment of the anatomical relationship between the arcuate eminence and superior semicircular canal by computed tomography. Neurol Med Chir (Tokyo). 2007;47(8):335-339; discussion 339-340.

6. Cömert E, Cömert A, Cay N, Tunçel U, Tekdemir I. Surgical Anatomy of the Infralabyrinthine Approach. Otolaryngol Head Neck Surg.
2014;151(2):301-307.

7. Mukherjee P, Uzun-Coruhlu H, Curthoys IS, Jones AS, Bradshaw AP, Pohl DV. Three-dimensional analysis of the vestibular end organs in relation to the stapes footplate and piston placement. Otol Neurotol. 2011;32(3):367-372.

8. Richard C, Laroche N, Malaval L, et al. New insight into the bony labyrinth: a microcomputed tomography study. Auris Nasus Larynx. 2010;37(2):155-161.

9. Connor SE, Bell DJ, O'Gorman R, Fitzgerald-O'Connor A. CT and MR imaging cochlear distance measurements may predict cochlear implant length required for a 360 degrees insertion. AJNR Am J Neuroradiol. 2009;30(7):1425-1430.

10. Memari F, Tabatabaee RM, Borghei Razavi H, Fereshtehnejad SM, Daneshi A. Transpetrosal approach: an anatomical study of temporal bone. Eur Arch Otorhinolaryngol. 2009;266(9):1373-1380.

11. Wysocki J, Skarzyñski H. Distances between the cochlea and adjacent structures related to cochlear implant surgery. Surg Radiol Anat. 1998;20(4):267-271.

12. Stjernholm C. Aspects of temporal bone anatomy and pathology in conjunction with cochlear implant surgery. Acta Radiol Suppl. 2003;430:2-15.

13. Atturo $F$, Barbara $M$, Rask-Andersen $H$. On the Anatomy of the 'Hook' Region of the Human Cochlea and How It Relates to Cochlear Implantation. Audiol Neurootol. 2014 Nov 4;19(6):378-385.

14. Choi BJ, Kim MJ, Chang KH, Yeo SW, Jun BC. Three-dimensional Anatomical Analysis of Surgical Landmarks for the Middle Cranial Fossa Approach. Indian J Otolaryngol Head Neck Surg. 2014;66(3):320-324.

15. Singla A, Sahni D, Gupta AK, Loukas M, Aggarwal A. Surgical anatomy of round window and its implications for cochlear implantation. Clin Anat. 2014;27(3):331-336

16. Yamanaka T, Pyle GM, Hosoi H. Transmastoid approach to the superior semicircular canal: An anatomical study. Auris Nasus Larynx. 2014;41(2):169-171.

17. Salma A1, Makiese $O$, Reiss A, Pillai $P$, Sammet $S$, Ammirati M. A microanatomical map of the structures hidden in the middle fossa based on the facial nerve hiatus: measurements and their variability. Clin Anat. 2013;26(4):436-443.

18. Bogar M, Bento RF, Tsuji RK. Cochlear anatomy study used to design surgical instruments for cochlear implants with two bundles of electrodes in ossified cochleas. Braz J Otorhinolaryngol. 2008;74 (2):194-199. 